\title{
Analytic theory of orbit contraction due to atmospheric drag $\dagger$
}

\author{
N. X. VINH AND J. M. LONGUSKI \\ The University of Michigan, Ann Arbor, MI 48109, U.S.A.
}

\author{
AND \\ A. BUSEMANN AND R. D. CULP \\ University of Colorado, Boulder, CO 80309, U.S.A.
}

(Received 14 June 1978; revised 4 October 1978)

\begin{abstract}
Abetract-The motion of a satellite in orbit, subject to atmospheric force and the motion of a reentry vehicle are governed by the same forces, namely, gravitational and aerodynamic. This sugsests the derivation of a uniform set of equations applicable to both cases.

For the case of satellite motion, by a proper transformation and by the method of averaging, a technique appropriate for long duration tight, the classical nonlinear differential equation describing the contraction of the major axis is derived. While previous authors, and in particular King-Hele, integrated this equation using various heuristic methods, the present authors present a rigorous analytic solution, with a high degree of accuracy, using Poincart's method of small parameters. Next, using Lagrange's expansion, the major axis is expressed explicitly as a function of the eccentricity. The solution is uniformly valid for moderate and small eccentricities. This is a major achievement due to the discovery of a certain recurrence formula which facilitates the long and tedious analytic process. For highly eccentric orbits, the asymptotic equation is derived directly from the general equation. To obtain the same equation King-Hele must use an entirely different method. Again, while King-Hele only succeeded in obtaining an approximate solution to this case using a heuristic method of integration, the exact solution to the asymptotic equation has been obtained by the present authors. Numerical solutions have been generated to display the accuracy of the analytic theory.

The explicit solution has been derived using a spherically symmetrical atmosphere with exponential variation of density with height but the basic equations developed and the tochnique for their integration apply to the case of an oblate atmosphere which is locally exponential.
\end{abstract}

\section{Introduction}

THE THEORY of satellite orbits in the presence of an atmosphere was developed during the late fifties with the launching of the first artificial satellite. With increasing knowledge of planetary atmospheres, especially the atmosphere of the Earth, the theory has now reached a high degree of elaboration. For a first estimation of the lifetime of a satellite and for a correlation between the semi-major axis and the eccentricity of the orbit while it undergoes a contraction due to the perturbing effect of atmospheric drag, analytic theory is adequate. The classical theory was presented in a monograph written by King-Hele (1964),

†Work supported by NASA Grant No. NSG-1448. 
who is among the authors who have contributed the most to the development of analytical solutions.

There are two reasons for presenting this new study of a well established and analyzed subject.

The first reason concerns the approach to this problem. In the early days, development of the theory of flight inside an atmosphere was conducted from two different approaches. On the one hand, researchers analyzed the small perturbations of satellite orbits at very high altitudes. The mathematical tools are perturbation theories in celestial mechanics based on Lagrange's equations for the variations of orbital elements. The space object, usually referred to as a satellite, is not intended for recovery. The main subjects of concern are its life expectancy and, while in orbit, the slow variations of its orbital elements. On the other hand, engineers and scientists who were concerned with the same recovery of an entry vehicle concentrated their effort in the study of the deceleration and heating during entry. Here the elements of prime consideration are the position and velocity of the vehicle, both varying rapidly. The smooth behavior of near Keplerian orbit is no longer valid and strong physical assumptions were made to such an extent that, although describing the same phenomenon, namely flight of an object inside a planetary atmosphere, the equations became totally different. The gap got wider, as the two theories became more and more sophisticated, so that now the two groups, one consisting mostly of mathematicians, and one consisting mostly of space dynamicists seldomly reference the other group's work. With the objective of providing a unified theory for flight inside a planetary atmosphere, we have formulated a set of universal, exact equations. These equations have been successfully applied to the study of planetary entry of a space vehicle (Vinh et al., 1977) and even to optimization of such an entry (Vinh et al., 1975). In this paper we shall present the necessary transformation such that the equations can be used for analyzing the slow variations of the orbital elements while the vehicle is still in near vacuum. This successful wedding is necessary since the future space vehicle is designed to stay for an extended period in orbit as a satellite, and also to be recovered safely after a fiery entry followed by a glide, an approach and a landing on an airfield.

The second reason concerns an improvement of the existing theory. Often, because of mathematical difficulties in the analytic integration of the equations of motion, the types of solutions are artificially classified. One can easily understand the classification of orbits into hyperbolic and elliptic because the natures of the orbits are different, as reflected by the Keplerian equations with $e>1$ and $e<1$. However, when it comes to different orbital phases with $e$ very small and with $e$ not so small, then it is clear that the classification is purely for easing the integration and usually the regions of validity of different solutions are at best defined empirically. Our effort in going over the classical theory is to remove, whenever possible, such an ambiguity.

Forces on a satellite in orbit

The satellite and the planet are assumed to be in two-body relative motion. For a spherical planet, the gravitational force is an inverse square force of 
attraction with acceleration

$$
g(\boldsymbol{r})=\frac{\mu}{r^{2}}
$$

The atmospheric force is in the form of drag, $D_{A}$, acting in a direction opposite to the velocity $v_{A}$ relative to the ambient air

$$
D_{A}=\frac{1}{2} \rho S C_{D} V_{A}^{2}
$$

$C_{D}$ is the drag coefficient measured using a reference area $S$ and the atmospheric density $\rho$. For the sake of mathematical purity of the theory, we shall use a strictly exponential law

$$
\rho=\rho_{p_{0}} \mathrm{e}^{\rho\left(r_{\rho_{0}}-r\right)}
$$

where $\beta$ is a constant

$$
\boldsymbol{\beta}=\frac{1}{\boldsymbol{H}}
$$

$H$ is the scale height, and subscript $p_{0}$ denotes the initial periapsis condition. Extension of the theory for including the effect of oblateness of the atmosphere will be discussed in the last section.

The equations of motion are written with respect to an inertial frame with the origin at the center of the planet. Let $\mathrm{V}$ be the absolute velocity of the satellite.

$$
\mathbf{v}=\mathbf{v}_{\mathbf{A}}+\mathbf{v}
$$

where $V_{e}$ is the velocity at the point $M$, of the ambient air relative to the planet center (Fig. 1). If $w$ is the angular velocity of the rotating atmosphere, then

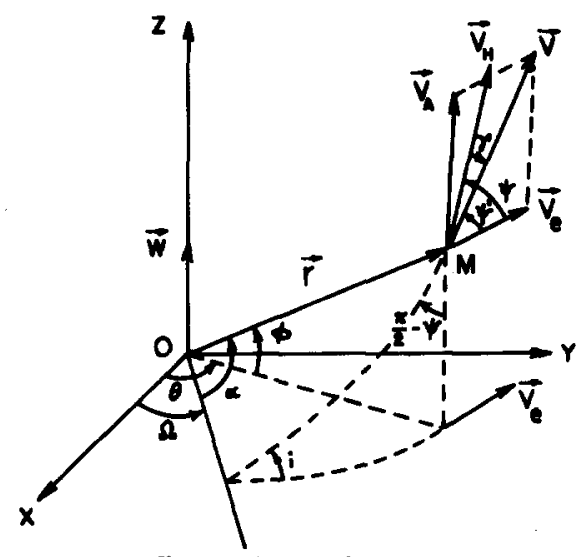

Fis. 1. Nomenclature. 


$$
V_{e}=r w \cos \phi
$$

where $\phi$ is the latitude of the point $M$.

Let $\psi^{\prime}$ be the angle between $V_{e}$ and $V$. Then by squaring eqn (5)

$$
V_{A}^{2}=V^{2}+V_{e}^{2}-2 V V_{e} \cos \psi^{\prime}
$$

The vector $V_{e}$ is in the local horizontal plane. Also, near the periapsis where the aerodynamic drag is most effective, the satellite travels in a nearly horizontal direction and hence the angle $\gamma$ between the velocity $\mathbf{V}$ and the horizontal plane is small. Then, the angle $\psi^{\prime}$ between $V_{e}$ and $V$ is nearly equal to the angle $\psi$ between $V_{e}$ and the projection $V_{H}$ of $V$ on the horizontal plane. This angle $\psi$, called the heading, is related to the latitude $\phi$ and the inclination $i$ of the orbital plane by the well-known relation

$$
\cos \psi \cos \phi=\cos i \text {. }
$$

Therefore, we have approximately

$$
V_{e} \cos \psi^{\prime} \approx V_{e} \cos \psi=r w \cos \phi \cos \psi=r w \cos i
$$

Upon substituting eqns (6) and (9) into eqn (7) we have

$$
V_{A}^{2}=V^{2}\left(1-\frac{r w}{V} \cos i\right)^{2}+r^{2} w^{2}\left(\cos ^{2} \phi-\cos ^{2} i\right)
$$

The rotation of the atmosphere is generally slow so that the term $w^{2}$ can be neglected. For the small term $r w / V$, it is appropriate to use an average value. King-Hele suggested using the value at perigee $r_{p_{0}} / V_{p_{0}}$ to replace $\gamma / V$. Finally $i$, which usually varies by less than $0.3^{\circ}$ during a satellite's life, may be taken equal to its initial value $i_{0}$. Then we have King-Hele's expression (King-Hele, 1964)

$$
V_{A}^{2}=f V^{2}
$$

where the average constant value $f$ is

$$
f=\left(1-\frac{r_{p_{0}} w}{V_{p_{0}}} \cos i_{0}\right)^{2}
$$

Thus, in terms of the absolute speed, the drag force is

$$
D_{A}=\frac{1}{2} \rho S f C_{D} V^{2}
$$

acting opposite to the direction of the velocity $V_{A}$ of the satellite relative to the ambient air. 


\section{The equations of motion}

For the flight of an aerodynamic vehicle with a lift coefficient $C_{L}$ and a drag coefficient $C_{D}$ it is customary to use the equations of motion with the notation in Fig. 1.

$$
\begin{aligned}
\frac{\mathrm{d} r}{\mathrm{~d} t} & =V \sin \gamma \\
\frac{\mathrm{d} \theta}{\mathrm{d} t} & =\frac{V \cos \gamma \cos \psi}{r \cos \phi} \\
\frac{\mathrm{d} \phi}{\mathrm{d} t} & =\frac{V \cos \gamma \sin \psi}{r} \\
\frac{\mathrm{d} V}{\mathrm{~d} t} & =-\frac{\rho S C_{D} V^{2}}{2 m}-g \sin \gamma \\
V \frac{\mathrm{d} \gamma}{\mathrm{d} t} & =\frac{\rho S C_{L} V^{2} \cos \sigma}{2 m}-\left(g-\frac{V^{2}}{r}\right) \cos \gamma \\
V \frac{\mathrm{d} \psi}{\mathrm{d} t} & =\frac{\rho S C_{L} V^{2} \sin \sigma}{2 m \cos \gamma}-\frac{V^{2}}{r} \cos \gamma \cos \psi \tan \phi
\end{aligned}
$$

where the bank angle $\sigma$ is defined as the angle between the local vertical plane containing the velocity and the plane containing the velocity and the aerodynamic force.

Using the dimensionless variables

$$
\begin{aligned}
& u=\frac{V^{2} \cos ^{2} \gamma}{g r} \\
& \bar{Z}=\frac{\rho S C_{D}}{2 m} \sqrt{\frac{r}{\beta}}
\end{aligned}
$$

and a dimensionless independent variable

$$
s=\int_{0}^{t}\left(\frac{V}{r}\right) \cos \gamma \mathrm{d} t
$$

we have derived the exact, universal equations for entry trajectories into a planetary atmosphere assumed to be at rest (Vinh et al., 1975)

$$
\begin{aligned}
& \frac{\mathrm{d} \bar{Z}}{\mathrm{~d} s}=-\beta r\left(-\frac{1}{\beta \rho} \frac{\mathrm{d} \rho}{\mathrm{d} r}-\frac{1}{2 \beta r}+\frac{1}{2 \beta^{2}} \frac{\mathrm{d} \beta}{\mathrm{d} r}\right) \bar{Z} \tan \gamma \\
& \frac{\mathrm{d} u}{\mathrm{~d} s}=-\frac{2 \sqrt{ }(\beta r) \bar{Z} u}{\cos \gamma}\left(1+\frac{C_{L}}{C_{D}} \cos \sigma \tan \gamma+\frac{\sin \gamma}{2 \sqrt{ }(\beta r) \bar{Z}}\right) \\
& \frac{\mathrm{d} \gamma}{\mathrm{d} s}=\frac{V(\beta r) \bar{Z}}{\cos \gamma}\left[\frac{C_{L}}{C_{D}} \cos \sigma+\frac{\cos \gamma}{\sqrt{ }(\beta r) \bar{Z}}\left(1-\frac{\cos ^{2} \gamma}{u}\right)\right]
\end{aligned}
$$




$$
\begin{aligned}
& \frac{\mathrm{d} \theta}{\mathrm{d} s}=\frac{\cos \psi}{\cos \phi} \\
& \frac{\mathrm{d} \phi}{\mathrm{d} s}=\sin \psi \\
& \frac{\mathrm{d} \psi}{\mathrm{d} s}=\frac{V(\beta r) \bar{Z}}{\cos ^{2} \gamma}\left(\frac{C_{L}}{C_{D}} \sin \sigma-\frac{\cos ^{2} \gamma \cos \psi \tan \phi}{\sqrt{ }(\beta r) \bar{Z}}\right)
\end{aligned}
$$

We shall use the necessary transformation to obtain the equations for satellite motion inside a rotating atmosphere.

First, still with the notion of an atmosphere at rest, we use the transformation

$$
\begin{aligned}
\cos \phi \cos \psi & =\cos i \\
\cos \phi \sin \psi & =\sin i \cos \alpha \\
\cos \alpha & =\cos \phi \cos (\theta-\Omega)
\end{aligned}
$$

to transform the last three equations of (17) into

$$
\begin{aligned}
& \frac{\mathrm{d} \alpha}{\mathrm{d} s}=1-\frac{\sqrt{ }(\beta r) \bar{Z} \sin \alpha}{\tan i \cos ^{2} \gamma}\left(\frac{C_{L}}{C_{D}}\right) \sin \sigma \\
& \frac{\mathrm{d} \Omega}{\mathrm{d} s}=\frac{\sqrt{ }(\beta r) \bar{Z} \sin \alpha}{\sin i \cos ^{2} \gamma}\left(\frac{C_{L}}{C_{D}}\right) \sin \sigma \\
& \frac{\mathrm{d} i}{\mathrm{~d} s}=\frac{\sqrt{ }(\beta r) \bar{Z} \cos \alpha}{\cos ^{2} \gamma}\left(\frac{C_{L}}{C_{D}}\right) \sin \sigma
\end{aligned}
$$

From Fig. 1 we notice that $i$ is the inclination and $\Omega$ the longitude of the ascending node of the osculating plane. The angle $\alpha$ is the angle between the ascending node and the position vector. The angle $\sigma$, as stated earlier, is the angle between the vertical plane passing through the velocity, the $(r, V)$ plane, and the plane containing the aerodynamic force and the velocity, the $(A, V)$ plane (Fig. 2). For satellite motion, we have a simplification, and at the same time, a complication. The simplification is that there is no lift. The complication is that the drag force is modified by the factor $f$ (eqn 12), and it is directed opposite to the velocity $V_{A}$, not the absolute velocity $V$.

Figure 2 is the aerodynamic force diagram used in deriving the equations of motion (14), to which we have added the velocity $v_{A}$ with respect to the ambient air, and the drag force $D_{A}$, opposite in direction to $V_{A}$. In the present situation, we remove the lift force $L$ and replace the vector drag $D$ by $D_{A}$. This force $D_{A}$ can be decomposed into one component in the orbital plane and one component normal to the orbital plane. Since $V_{e}$ is small, $V_{A}$ is nearly aligned to $V$ and the drag component in the orbital plane can be considered as directly opposite to $V$, with magnitude $D_{A}$ as given by eqn (13). To have the component $D_{N}$ of $D_{A}$ 


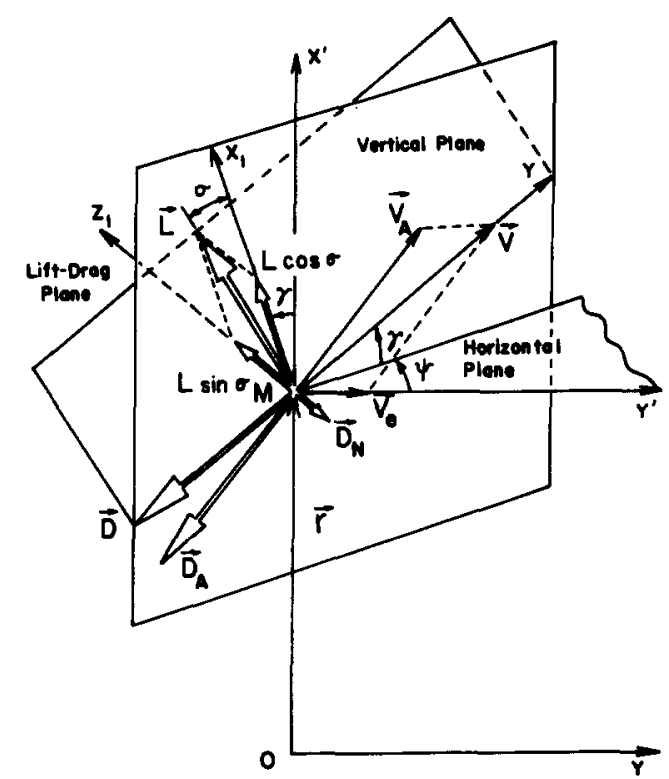

Fig. 2. Aerodynamic forces.

orthogonal to the orbital plane we find the projection of

$$
\mathrm{D}_{A}=-\frac{1}{2} \rho S f C_{D} V^{2} \frac{\mathbf{V}_{A}}{V_{A}}
$$

By the vector relation (5), since $V$ is in the orbital plane, and since $V$, makes an angle $\psi$ with the orbital plane, the projection of $V_{A}$ on the normal to the orbital plane is the same as the projection of $V_{e}$ which has magnitude

$$
V_{e} \sin \psi=r w \cos \phi \sin \psi=r w \sin i \cos \alpha .
$$

Hence, the vector $D_{N}$ has magnitude

$$
D_{N}=\frac{1}{2} \rho S f C_{D} r w \sin i \cos \alpha \frac{V^{2}}{V_{A}}
$$

By relation (11), we write it as

$$
D_{N}=\frac{\rho S f C_{D} V}{2 f^{1 / 2}} r w \sin i \cos \alpha
$$

and its direction is opposite to the vector $L \sin \sigma$ in Fig. 2.

The end result of the analysis is that, in the eqns (17) and (19), we replace $C_{D}$ by the modified drag coefficient $f C_{D}$, we delete the component $C_{L} \cos \sigma$ and replace the component $C_{\mathrm{L}} \sin \sigma$ by

$$
C_{L} \sin \sigma=-f^{1 / 2} C_{D}\left(\frac{r w}{V}\right) \sin i \cos \alpha
$$


Finally, the variable $\bar{Z}$, the modified Chapman $\bar{Z}$ function defined by eqn (15), is most effective in analyzing the entry phase of the vehicle. While the vehicle is still in orbit, we use it in the form

$$
V(\beta r) \bar{Z}=\bar{Z}_{0}\left(\frac{r}{r_{p_{0}}}\right) \mathrm{e}^{\beta\left(r_{p_{0}}-r\right)}
$$

where the dimensionless constant $\overline{\bar{Z}}_{0}$ is

$$
\bar{Z}_{0}=\frac{\rho_{p_{0}} S f C_{D}^{r_{p_{0}}}}{2 m}
$$

We can now rewrite the eqns (17) and (19), introducing the equation for $H r_{p_{0}}$ to replace the equation for $\bar{Z}$

$$
\begin{aligned}
\frac{\mathrm{d}}{\mathrm{d} s}\left(\frac{r}{r_{p_{0}}}\right) & =\left(\frac{r}{r_{p_{0}}}\right) \tan \gamma \\
\frac{\mathrm{d} u}{\mathrm{~d} s} & =-u \tan \gamma-\frac{2 \bar{Z}_{0} u}{\cos \gamma}\left(\frac{r}{r_{p_{0}}}\right) \mathrm{e}^{\beta\left(r_{p_{0}}-r\right)} \\
\frac{\mathrm{d} \gamma}{\mathrm{d} s} & =1-\frac{\cos ^{2} \gamma}{u} \\
\frac{\mathrm{d} \alpha}{\mathrm{d} s} & =1+\frac{r_{p_{0}} w \bar{Z}_{0}}{\sqrt{ }\left(\mu f / r_{p_{0}}\right)}\left(\frac{r}{r_{p_{0}}}\right)^{5 / 2} \frac{\cos i \sin \alpha \cos \alpha}{u^{1 / 2} \cos \gamma} \mathrm{e}^{\beta\left(r_{p_{0}}-r\right)} \\
\frac{\mathrm{d} \Omega}{\mathrm{d} s} & =-\frac{r_{p_{0}} w \bar{Z}_{0}}{\sqrt{ }\left(\mu f / r_{p_{0}}\right)}\left(\frac{r}{r_{p_{0}}}\right)^{5 / 2} \frac{\sin \alpha \cos \alpha}{u^{1 / 2} \cos \gamma} \mathrm{e}^{\beta\left(r_{p_{0}}-r\right)} \\
\frac{\mathrm{d} i}{\mathrm{~d} s} & =-\frac{r_{p_{0}} w \bar{Z}_{0}}{\sqrt{ }\left(\mu f / r_{p_{0}}\right)}\left(\frac{r}{r_{p_{0}}}\right)^{5 / 2} \frac{\sin i \cos \alpha}{u^{1 / 2} \cos \gamma} \mathrm{e}^{\beta\left(r_{p_{0}}-r\right)} .
\end{aligned}
$$

The variational equations

Equations (27) are the bridge between satellite theory and entry theory. As a matter of fact, they can be used to follow the motion of a vehicle subject to gravitational force and drag force of a rotating planet for its entire life in orbit until the end of its entry and contact with the planetary surface. The accuracy depends on the readjustment, for each layer of the atmosphere, of the "constant value" $\beta$. The equations are most useful for analyzing the last few revolutions and the entry phase. The variables $\alpha, \Omega$ and $i$ which are orbital elements are related to the entry elements $\phi, \theta$ and $\psi$ through the relations (18). On the other hand, the variables $r, u$ and $\gamma$ which are the entry variables can be transformed into the orbital elements through explicit relations.

Consider the osculating orbit, that is, the orbit the vehicle would follow if at any time the drag force suddenly vanished. Putting $\bar{Z}_{0}=0$ in eqns (27), we have 


$$
\begin{aligned}
\frac{\mathrm{d}}{\mathrm{d} s}\left(\frac{r}{r_{p_{0}}}\right) & =\left(\frac{r}{r_{p_{0}}}\right) \tan \gamma \\
\frac{\mathrm{d} u}{\mathrm{~d} s} & =-u \tan \gamma \\
\frac{\mathrm{d} \gamma}{\mathrm{d} s} & =1-\frac{\cos ^{2} \gamma}{u} \\
\frac{\mathrm{d} \alpha}{\mathrm{d} s} & =1 \\
\frac{\mathrm{d} \Omega}{\mathrm{d} s} & =0 \\
\frac{\mathrm{d} i}{\mathrm{~d} s} & =0 .
\end{aligned}
$$

The integration is simple and we have the general solution

$$
\begin{aligned}
\cos ^{2} \gamma & =\frac{u^{2}}{2 u-C_{1}} \\
r & =\frac{C_{2}}{u} \\
u & =1+\sqrt{ }\left(1-C_{1}\right) \cos \left(s-C_{3}\right) \\
s & =\alpha+C_{4} \\
\Omega & =C_{5} \\
i & =C_{6} .
\end{aligned}
$$

We see that $s$ is equivalent to $\alpha$ and actually, we have only 5 constants of integration. The last constant of integration is obtained through the time equation (eqn 16). In the first three equations, we evaluate the constants of integration by taking the origin of time at the time of passage through the periapsis. We have

$$
\begin{aligned}
\cos ^{2} \gamma & =\frac{u^{2}}{2 u-\left(1-e^{2}\right)} \\
u & =1+e \cos (\alpha-\omega) \\
r & =\frac{a\left(1-e^{2}\right)}{1+e \cos (\alpha-\omega)}
\end{aligned}
$$

These three equations provide the link between the entry variables $r, u$ and $\gamma$ and the semi-major axis $a$, the eccentricity $e$ and the argument of the periapsis $\omega$, which are the orbital elements used in the theory of orbits.

During the phase in orbit, $\bar{Z}_{0}$ is small and the orbital elements vary slowly. By 
taking the derivatives of eqns (30), considering $a, e$ and $\omega$ as varying quantities and using the eqns (27) for the derivatives of $r, u$ and $\gamma$ we have the variational equations for $a, e$ and $\omega$.

First, for $e$, we have

$$
\frac{\mathrm{d} e}{\mathrm{~d} s}=\frac{2 \bar{Z}_{0} u^{2}}{e \cos ^{3} \gamma}\left(\frac{\cos ^{2} \gamma}{u}-1\right)\left(\frac{r}{r_{p_{0}}}\right) \mathrm{e}^{\beta\left(r_{p_{0}}-r\right)}
$$

We present the equation in this form to show an interesting behavior of the eccentricity of the osculating orbit. It is a general belief that the eccentricity decreases continuously under the action of atmospheric drag. This, however, is only the secular effect. During each revolution, the flight path angle passes through a maximum and a minimum as seen by the third equation of (27), and by eqn (31) it is seen that, at the same time, the eccentricity passes through a minimum and a maximum respectively.

Next, we shall use the more familiar eccentric anomaly $E$ to replace $s$ as an independent variable in the variational equations. The following relations can be easily derived.

$$
\begin{aligned}
\frac{r}{a} & =\frac{\left(1-e^{2}\right)}{1+e \cos (\alpha-\omega)}=1-e \cos E \\
u & =\frac{\left(1-e^{2}\right)}{1-e \cos E} \\
\cos ^{2} \gamma & =\frac{\left(1-e^{2}\right)}{(1-e \cos E)(1+e \cos E)} \\
\frac{\mathrm{d} s}{\mathrm{~d} E} & =\frac{\sqrt{ }\left(1-e^{2}\right)}{1-e \cos E} .
\end{aligned}
$$

Hence, changing the independent variable from $s$ to $E$, the equation for $e$ has the form

$$
\frac{\mathrm{d} e}{\mathrm{~d} E}=-2 \bar{Z}_{0}\left(1-e^{2}\right)\left(\frac{a}{r_{p_{0}}}\right) \cos E\left(\frac{1+e \cos E}{1-e \cos E}\right)^{1 / 2} e^{\beta\left(r_{p_{0}}-r\right)}
$$

Similarly, we have for the semi-major axis

$$
\frac{\mathrm{d} a}{\mathrm{~d} E}=-2 \bar{Z}_{0} \frac{a^{2}}{r_{p_{0}}} \frac{(1+e \cos E)^{3 / 2}}{(1-e \cos E)^{1 / 2}} \mathrm{e}^{\beta\left(r_{p_{0}}-r\right)}
$$

The last three equations of (27) become

$$
\begin{aligned}
\frac{\mathrm{d} \alpha}{\mathrm{d} E}-\frac{\mathrm{d} \omega}{\mathrm{d} E}= & \frac{V\left(1-e^{2}\right)}{1-e \cos E}\left[1+\frac{2 \bar{Z}_{0}}{e}\left(\frac{a}{r_{p_{0}}}\right) \sin E(1-e \cos E)^{1 / 2}\right. \\
& \left.\times(1+e \cos E)^{1 / 2} e^{\beta\left(r_{p_{0}}-r\right)}\right]
\end{aligned}
$$




$$
\begin{aligned}
\frac{\mathrm{d} \Omega}{\mathrm{d} E}= & -\frac{r_{p_{0}} w \bar{Z}_{0}}{\sqrt{ }\left(\mu f / r_{p_{0}}\right) \sqrt{ }\left(1-e^{2}\right)}\left(\frac{a}{r_{p_{0}}}\right)^{5 / 2}(1-e \cos E)^{5 / 2}(1+e \cos E)^{1 / 2} \\
& \times \sin \alpha \cos \alpha \mathrm{e}^{\beta\left(r_{p_{0}}-r\right)} \\
\frac{\mathrm{d} i}{\mathrm{~d} E}= & -\frac{r_{p_{0}} w \bar{Z}_{0}}{\sqrt{ }\left(\mu f / r_{p_{0}}\right) \sqrt{ }\left(1-e^{2}\right)}\left(\frac{a}{r_{p_{0}}}\right)^{5 / 2}(1-e \cos E)^{5 / 2}(1+e \cos E)^{1 / 2} \\
& \times \sin i \cos ^{2} \alpha \mathrm{e}^{\beta\left(r_{p_{0}}-r\right)} .
\end{aligned}
$$

The contraction of orbits

The average equation

Consider the variations in $e$ and $a$, eqns (33) and (34). Under the dissipative effect of the drag, the major axis decreases while the eccentricity, although having an oscillatory behavior, also decreases secularly with the time.

For the radial distance we have,

$$
\begin{aligned}
r & =a(1-e \cos E) \\
r_{p_{0}} & =a_{0}\left(1-e_{0}\right) .
\end{aligned}
$$

We write the exponential function in the equations

$$
\exp \left[\beta\left(r_{D_{0}}-r\right)\right]=\exp \left[\beta\left(a_{0}-a-a_{0} e_{0}\right)+\beta a e \cos E\right]
$$

Along each revolution $a$ is nearly constant while the varying quantity $\beta a e \cos E$ provides the fluctuation in atmospheric density. This leads to a natural choice of the dimensionless variable

$$
x=\beta a e
$$

to replace the eccentricity. The equation for $e$ is replaced by

$$
\frac{\mathrm{d} x}{\mathrm{~d} E}=-\frac{2 \bar{Z}_{0} \beta a^{2}}{r_{p_{0}}}(e+\cos E)\left(\frac{1+e \cos E}{1-e \cos E}\right)^{1 / 2} \mathrm{e}^{\beta\left(r_{p_{0}}-r\right)}
$$

The new variable $x$ behaves like the eccentricity $e$; that is, during each revolution $x$ passes through stationary values when $\cos E=-e$. On the average, however, $x$ decreases with the time. Since the decaying process is slow we can use the averaging technique (Bogoliubov and Mitropolsky, 1961), applied to the right hand side of the eqns (34) and (39) for $a$ and $x$.

For the equation for $a$, we have

$$
\frac{\mathrm{d} a}{\mathrm{~d} E}=-2 \bar{Z}_{0} \frac{a^{2}}{r_{p_{0}}} \exp \left[\beta\left(a_{0}-a-a_{0} e_{0}\right)\right] \frac{1}{2 \pi} \int_{0}^{2 \pi} \frac{(1+e \cos E)^{3 / 2}}{(1-e \cos E)^{1 / 2}} \exp (x \cos E) \mathrm{d} E .
$$


For small eccentricity, the integrand can be expanded in a power series in $e$ and upon integrating, we have

$$
\begin{aligned}
\frac{\mathrm{d} a}{\mathrm{~d} E}= & -\frac{2 \bar{Z}_{0} a^{2}}{r_{p_{0}}} \exp \left[\beta\left(a_{0}-a-a_{0} e_{0}\right)\right] \\
& \times\left\{I_{0}+2 e I_{1}+\frac{3}{4} e^{2}\left(I_{0}+I_{2}\right)+\frac{e^{3}}{4}\left(3 I_{1}+I_{3}\right)+\frac{e^{4}}{64}\left(21 I_{0}+28 I_{2}+7 I_{4}\right)+0\left(e^{3}\right)\right\}
\end{aligned}
$$

where $I_{n}(x)$ is the Bessel function of the first kind and of imaginary argument, of order $n$

$$
I_{n}(x)=\frac{1}{2 \pi} \int_{0}^{2 \pi} \cos n E \exp (x \cos E) \mathrm{d} E
$$

Similarly, the average equation for $x$ is

$$
\begin{aligned}
\frac{\mathrm{dx}}{\mathrm{d} E}= & \frac{-2 \bar{Z}_{0} \beta a^{2}}{r_{p_{0}}} \exp \left[\beta\left(a_{0}-a-a_{0} e_{0}\right)\right]\left\{I_{1}+\frac{1}{2} e\left(3 I_{0}+I_{2}\right)+\frac{e^{2}}{8}\left(11 I_{1}+I_{3}\right)\right. \\
& \left.+\frac{e^{3}}{16}\left(7 I_{0}+8 I_{2}+I_{4}\right)+\frac{e^{4}}{128}\left(78 I_{1}+31 I_{3}+3 I_{5}\right)+0\left(e^{5}\right)\right\}
\end{aligned}
$$

Let

$$
Z=\frac{a}{a_{0}}
$$

be the dimensionless semi-major axis. By dividing the eqn (41) by eqn (43) and expanding the ratio in a power series in $e$ we have

$$
\begin{aligned}
\beta a_{0} \frac{\mathrm{d} Z}{\mathrm{~d} x}= & y_{0}+\frac{1}{2} e\left(4-3 y_{0}^{2}-y_{0} y_{2}\right)+\frac{1}{8} e^{2}\left[2 y_{0}\left(3 y_{0}+y_{2}\right)^{2}-29 y_{0}-2 y_{2}-y_{0} y_{3}\right] \\
& +\frac{e^{3}}{16}\left[-32+113 y_{0}^{2}+38 y_{0} y_{2}-y_{0} y_{4}+2 y_{2}^{2}+6 y_{0}^{2} y_{3}+2 y_{0} y_{2} y_{3}\right. \\
& \left.-2 y_{0}\left(3 y_{0}+y_{2}\right)^{3}\right] \\
& +\frac{e^{4}}{128}\left[8 y_{0}\left(3 y_{0}+y_{2}\right)^{4}-8\left(3 y_{0}+y_{2}\right)^{2}\left(9 y_{0}+y_{2}\right)-12 y_{0}\left(3 y_{0}+y_{2}\right)^{2}\left(11+y_{3}\right)\right. \\
& +2 y_{0}\left(11+y_{3}\right)^{2}+8 y_{0}\left(3 y_{0}+y_{2}\right)\left(7 y_{0}+8 y_{2}+y_{4}\right)+16\left(3 y_{0}+y_{2}\right)\left(19+y_{3}\right) \\
& \left.-12\left(y_{0}+y_{2}\right)\left(11+y_{3}\right)-y_{0}\left(78+31 y_{3}+3 y_{5}\right)-2\left(35 y_{0}+36 y_{2}+y_{4}\right)\right]+0\left(e^{5}\right)
\end{aligned}
$$

where we have defined the ratios of Bessel functions

$$
y_{n}=\frac{I_{n}}{I_{1}}, \quad n \neq 1
$$


The equation (45) has been derived by King-Hele, to the order $e^{3}$. For $x>3$ he integrated this equation by using the asymptotic expansions of the function $y_{n}(x)$. In this case the right hand side of eqn (45) has a very simple form and the semi-major axis $a$ is obtained by quadrature. Mathematically, the method of integration is not rigorous since on the right hand side of eqn (45), the eccentricity is a function of $a$ and $x$ by the definition (38); hence, the equation is actually a nonlinear equation in $a$. We shall arrange the equation in a form that allows Poincarés method of perturbations to be applied (Poincaré, 1960).

The Bessel functions satisfy the recurrence formula

$$
I_{n-1}(x)-I_{n+1}(x)=\frac{2 n}{x} I_{n}(x)
$$

so that any $y_{n}(x)$ can be expressed in terms of $y_{0}(x)$ and $x$. For example

$$
\begin{aligned}
& y_{2}=y_{0}-\frac{2}{x} \\
& y_{3}=1+\frac{8}{x^{2}}-\frac{4}{x} y_{0} \\
& y_{4}=\frac{-8}{x}-\frac{48}{x^{3}}+y_{0}+24 \frac{y_{0}}{x^{2}} \\
& y_{5}=1+\frac{72}{x^{2}}+\frac{384}{x^{4}}-\frac{12}{x} y_{0}-\frac{192}{x^{3}} y_{0} .
\end{aligned}
$$

Next, the eccentricity e, expressed in terms of $Z$ and $x$ is

$$
e=\epsilon \frac{x}{Z}
$$

where

$$
\epsilon=\frac{1}{\beta a_{0}}
$$

is a small quantity of the order $10^{-2}$ or less. Then, we can write eqn (45)

$$
\begin{aligned}
\frac{\mathrm{d} Z}{\mathrm{~d} x}= & \epsilon y_{0}+\epsilon^{2} \frac{x}{Z}\left(2-2 y_{0}{ }^{2}+\frac{y_{0}}{x}\right)+\epsilon^{3} \frac{x^{2}}{2 Z^{2}}\left(-8 y_{0}-7 \frac{y_{0}^{2}}{x}+8 y_{0}{ }^{3}+\frac{1}{x}\right) \\
& +\epsilon^{4} \frac{x^{3}}{2 Z^{3}}\left(-4+20 y_{0}{ }^{2}-10 \frac{y_{0}}{x}+4 \frac{y_{0}}{x^{3}}-5 \frac{y_{0}^{2}}{x^{2}}+20 \frac{y_{0}^{3}}{x}-16 y_{0}{ }^{4}+\frac{1}{x^{2}}\right) \\
& +\epsilon^{5} \frac{x^{4}}{4 Z^{4}}\left(32 y_{0}-96 y_{0}^{3}+82 \frac{y_{0}^{2}}{x}-\frac{6}{x}-17 \frac{y_{0}}{x^{2}}+\frac{3}{x^{3}}-24 \frac{y_{0}{ }^{2}}{x^{3}}\right. \\
& \left.+49 \frac{y_{0}^{3}}{x^{2}}-16 \frac{y_{0}}{x^{4}}-104 \frac{y_{0}^{4}}{x}+64 y_{0}^{5}\right)+0\left(\epsilon^{6}\right) .
\end{aligned}
$$


We see that the true nature of the equation is a nonlinear equation. Since $\epsilon$ is very small we need not go further with the expansion, and to the order $\epsilon^{5}$ included, the solution of this equation can be considered as the exact solution of eqn (45) truncated to the order $e^{4}$ included. We shall use Poincare's method of small parameters for the integration of eqn (51).

\section{Integration by Poincare's method of small parameters}

Poincarés method for integration (Poincaré, 1960) of a nonlinear differential equation containing a small parameter is a rigorous mathematical technique, proven to be convergent for small values of the parameter $\epsilon$. It has been used extensively in analytic work in celestial mechanics (Moulton, 1920).

We assume a solution for $Z$ of the form

$$
Z=Z_{0}+\epsilon Z_{1}+\epsilon^{2} Z_{2}+\epsilon^{3} Z_{3}+\epsilon^{4} Z_{4}+\epsilon^{3} Z_{5}+\cdots
$$

Upon substituting into eqn (51) and equating coefficients of like powers in $\epsilon$, we have the equations for the $Z_{i}$

$$
\begin{aligned}
& \frac{\mathrm{d} Z_{0}}{\mathrm{~d} x}=0 \\
& \frac{\mathrm{d} Z_{1}}{\mathrm{~d} x}=y_{0} \\
& \frac{\mathrm{d} Z_{2}}{\mathrm{~d} x}=\frac{x}{Z_{0}}\left(2+\frac{y_{0}}{x}-2 y_{0}^{2}\right) \\
& \frac{\mathrm{d} Z_{3}}{\mathrm{~d} x}=\frac{-x Z_{1}}{Z_{0}^{2}}\left(2+\frac{y_{0}}{x}-2 y_{0}^{2}\right)+\frac{x^{2}}{2 Z_{0}^{2}}\left(\frac{1}{x}-8 y_{0}-7 \frac{y_{0}^{2}}{x}+8 y_{0}^{3}\right) \\
& \frac{\mathrm{d} Z_{4}}{\mathrm{~d} x}=\frac{-x^{2} Z_{1}}{Z_{0}^{3}}\left(\frac{1}{x}-8 y_{0}-7 \frac{y_{0}^{2}}{x}+8 y_{0}^{3}\right)+\frac{x}{Z_{0}}\left(2+\frac{y_{0}}{x}-2 y_{0}{ }^{2}\right)\left(\frac{Z_{1}^{2}}{Z_{0}^{2}}-\frac{Z_{2}}{Z_{0}}\right) \\
& +\frac{x^{3}}{2 Z_{0}^{3}}\left(-4+\frac{1}{x^{2}}+20 y_{0}^{2}-10 \frac{y_{0}}{x}+4 \frac{y_{0}}{x^{3}}-5 \frac{y_{0}^{2}}{x^{2}}+20 \frac{y_{0}^{3}}{x}-16 y_{0}^{4}\right) \\
& \frac{\mathrm{d} Z_{5}}{\mathrm{~d} x}=\frac{x}{Z_{0}}\left(2 \frac{Z_{1} Z_{2}}{Z_{0}^{2}}-\frac{Z_{1}^{3}}{Z_{0}^{3}}-\frac{Z_{3}}{Z_{0}}\right)\left(2-2 y_{0}{ }^{2}+\frac{y_{0}}{x}\right) \\
& +\frac{x^{2}}{Z_{0}^{2}}\left(\frac{3}{2} \frac{Z_{1}^{2}}{Z_{0}^{2}}-\frac{Z_{2}}{Z_{0}}\right)\left(-8 y_{0}-7 \frac{y_{0}^{2}}{x}+8 y_{0}^{3}+\frac{1}{x}\right) \\
& -\frac{3 x^{3} Z_{1}}{2 Z_{0}^{4}}\left(-4+20 y_{0}^{2}-10 \frac{y_{0}}{x}+4 \frac{y_{0}}{x^{3}}-5 \frac{y_{0}^{2}}{x^{2}}+20 \frac{y_{0}^{3}}{x}-16 y_{0}^{4}+\frac{1}{x^{2}}\right) \\
& +\frac{x^{4}}{4 Z_{0}}\left(32 y_{0}-96 y_{0}{ }^{3}+82 \frac{y_{0}^{2}}{x}-\frac{6}{x}-17 \frac{y_{0}}{x^{2}}+\frac{3}{x^{3}}-24 \frac{y_{0}^{2}}{x^{3}}\right. \\
& \left.+49 \frac{y_{0}^{3}}{x^{2}}-16 \frac{y_{0}}{x^{4}}-104 \frac{y_{0}^{4}}{x}+64 y_{0}^{5}\right)
\end{aligned}
$$

with the initial conditions 


$$
Z_{0}\left(x_{0}\right)=1, \quad Z_{1}\left(x_{0}\right)=Z_{2}\left(x_{0}\right)=\cdots=0 .
$$

The integrations of eqns (53) is accomplished by successive quadratures and its success depends on whether or not the integrals can be expressed in terms of known functions. It has been found that the following recurrence formula is very useful. We have

$$
\int p(x) y_{0}{ }^{n+1} \mathrm{~d} x=-\frac{p(x)}{n} y_{0}{ }^{n}+\int p(x) y_{0}{ }^{n-1} \mathrm{~d} x+\int\left[\frac{p(x)}{x}+\frac{p^{\prime}(x)}{n}\right] y_{0}{ }^{n} \mathrm{~d} x
$$

where $n \neq 0$ and $p(x)$ is any arbitrary function. To derive the formula we use the well-known relation

$$
x I_{n}^{\prime}(x)+n I_{n}(x)=x I_{n-1}(x)
$$

so that for $n=1$

$$
y_{0}=\frac{I_{1}^{\prime}}{I_{1}}+\frac{1}{x}
$$

and for $n=0$

$$
I_{0}^{\prime}=I_{1}(x)
$$

Therefore, if $y_{0}=I_{0} / I_{1}$

$$
y_{0}^{\prime}=\frac{I_{0}^{\prime}}{I_{1}}-\frac{I_{0} I_{1}^{\prime}}{I_{1}^{2}}=1+\frac{y_{0}}{x}-y_{0}^{2}
$$

Now consider

or

$$
\int p(x) \mathrm{d}\left(\frac{y_{0}{ }^{n}}{n}\right)=\frac{p(x)}{n} y_{0}{ }^{n}-\int \frac{p^{\prime}(x)}{n} y_{0}{ }^{n} \mathrm{~d} x
$$

$$
\begin{aligned}
\int p(x) y_{0}^{n-1} y_{0}^{\prime} \mathrm{d} x & =\int p(x) y_{0}{ }^{n-1}\left(1+\frac{y_{0}}{x}-y_{0}^{2}\right) \mathrm{d} x \\
& =\frac{p(x)}{n} y_{0}{ }^{n}-\int \frac{p^{\prime}(x)}{n} y_{0}{ }^{n} \mathrm{~d} x .
\end{aligned}
$$

Rearranging the equation, we have the recurrence formula (55).

Using these relations, we proceed with the integrations of the eqn (53) using the initial conditions (54).

We have

and by eqn (57)

$$
Z_{0}(x)=1
$$

$$
Z_{1}=\log \frac{x I_{1}(x)}{x_{0} I_{1}\left(x_{0}\right)}
$$

where $x_{0}=\beta a_{0} e_{0}$ is the initial value of $x$. 
With $Z_{0}=1$, the equation for $Z_{2}$ is

$$
\frac{d Z_{2}}{d x}=2 x+y_{0}-2 x y_{0}^{2}
$$

Integrating

$$
Z_{2}=x^{2}+\log x I_{1}(x)-2 \int x y_{0}^{2} \mathrm{~d} x
$$

By the recurrence formula (55) with $p(x)=x, n=1$

$$
\int x y_{0}^{2} \mathrm{~d} x=\frac{1}{2} x^{2}-x y_{0}+2 \log x I_{1}(x)
$$

so that, in eqn (62) with the initial conditions, we have

$$
Z_{2}=2 x y_{0}(x)-2 x_{0} y_{0}\left(x_{0}\right)-3 \log \frac{x I_{1}(x)}{x_{0} I_{1}\left(x_{0}\right)}
$$

The integrations for obtaining $Z_{3}, Z_{4}$ and $Z_{5}$ are performed the same way, but they are much more laborious. It is found that the $Z_{l}(x)$ can be expressed in terms of two functions

$$
A(x)=x \frac{I_{0}(x)}{I_{1}(x)}=x y_{0}(x), \quad Z_{1}(x)=\log \frac{x I_{1}(x)}{x_{0} I_{1}\left(x_{0}\right)}
$$

We have the final solution

$$
\begin{aligned}
Z_{0}(x)= & 1 \\
Z_{1}(x)= & \log \frac{x I_{1}(x)}{x_{0} I_{1}\left(x_{0}\right)} \\
Z_{2}(x)= & 2\left(A-A_{0}\right)-3 Z_{1} \\
Z_{3}(x)= & \frac{7}{2}\left(x^{2}-x_{0}^{2}\right)-\frac{13}{2}\left(A-A_{0}\right)-2\left(A^{2}-A_{0}^{2}\right)+13 Z_{1}-2 A Z_{1}+\frac{3}{2} Z_{1}^{2} \\
Z_{4}(x)= & -\frac{35}{2}\left(x^{2}-x_{0}^{2}\right)+\frac{71}{2}\left(A-A_{0}\right)+3\left(A^{2}-A_{0}^{2}\right)+\frac{8}{3}\left(A^{3}-A_{0}^{3}\right) \\
& +4 A_{0}\left(A-A_{0}\right)-2\left(x^{2} A-x_{0}^{2} A_{0}\right) \\
& -\left(69+6 A_{0}+7 x^{2}-19 A-4 A^{2}\right) Z_{1}-\frac{35}{2} Z_{1}^{2} \\
& -Z_{1}^{3}+2 A Z_{1}^{2}
\end{aligned}
$$




$$
\begin{aligned}
Z_{5}(x)= & Z_{1}^{2}\left(162+6 A_{0}\right)+\frac{41}{2} Z_{1}^{3}+\frac{3}{4} Z_{1}^{4} \\
& +Z_{1}\left(437-\frac{21}{2} x_{0}^{2}+\frac{143}{2} A_{0}+6 A_{0}^{2}\right)-2 Z_{1}^{3} A-6 Z_{1}^{2} A^{2} \\
& -\frac{69}{2} Z_{1}^{2} A+\frac{21}{2} Z_{1}^{2} x^{2}-8 A^{3} Z_{1}-21 A^{2} Z_{1}+6 x^{2} A Z_{1} \\
& +A Z_{1}\left(\frac{-343}{2}-8 A_{0}\right)+\frac{147}{2} x^{2} Z_{1} \\
& +\frac{3}{4}\left(x^{4}-x_{0}^{4}\right)+\left(14 A_{0}+\frac{885}{8}\right)\left(x^{2}-x_{0}{ }^{2}\right) \\
& +\left(7 x_{0}^{2}-39 A_{0}-4 A_{0}^{2}-\frac{441}{2}\right)\left(A-A_{0}\right) \\
& -\frac{23}{2} x^{2} A+\frac{23}{2} x_{0}^{2} A_{0}+\left(\frac{-97}{8}-8 A_{0}\right)\left(A^{2}-A_{0}{ }^{2}\right) \\
& +4 x^{2} A^{2}-4 x_{0}^{2} A_{0}^{2}+2 A^{3}-2 A_{0}^{3}-4 A^{4}+4 A_{0}^{4} .
\end{aligned}
$$

The semi-major axis of the orbit under contraction is

$$
\frac{a}{a_{0}}=1+\epsilon Z_{1}+\epsilon^{2} Z_{2}+\epsilon^{3} Z_{3}+\epsilon^{4} Z_{4}+\epsilon^{5} Z_{5}
$$

Using $x$ as a parameter, we easily express the other quantities of interest. The eccentricity $e$ is given by eqn (49) while the drop in the periapsis is obtained from

$$
\frac{r_{p_{0}}-r_{p}}{H}=\beta\left[r_{p_{0}}-a(1-e)\right]=\beta a_{0}-\beta a_{0} e_{0}+\beta a e-\beta a_{0}\left(1+\epsilon Z_{1}+\cdots\right)
$$

or

$$
\frac{r_{p_{0}}-r_{p}}{H}=\left(x-x_{0}\right)-\left(Z_{1}+\epsilon Z_{2}+\epsilon^{2} Z_{3}+\epsilon^{3} Z_{4}+\epsilon^{4} Z_{5}\right)
$$

The ratio of the eccentricity can be obtained from

$$
\frac{e}{e_{0}}=\frac{1}{Z}\left(\frac{x}{x_{0}}\right)
$$

For each initial value $\epsilon=H / a_{0}$, and eccentricity $e_{0}$, we can calculate the initial value $x_{0}=\beta a_{0} e_{0}=e_{0} / \epsilon$ and evaluate the expressions for $a / a_{0}, e / e_{0}$ and $\left(r_{p_{0}}-r_{p}\right) / H$ as a function of $x$. Then these functions can be cross plotted in any combination.

The orbital period is

$$
\frac{T}{T_{0}}=\left(\frac{a}{a_{0}}\right)^{3 / 2}=(Z(x))^{3 / 2}
$$


Since the quantities $a, e, r_{p}$ and $T$ are all easily observable, and the integration has been performed to $e^{4}$ included, for small and moderate eccentricity, which is the case for most scientific Earth satellites, the equations can be used to verify the assumption made on the atmosphere. In general, the density, as a first approximation, can be assumed to be locally exponential. That is to say, the parameter $\beta$, or $H=1 / \beta$, can be assumed constant for each layer of the atmosphere. Since the value of $\beta$ enters the analytic solution, by adjusting for concordance between theory and observation, $\beta$ can be determined.

The expansions used to obtain the basic nonlinear equation (51) are only valid for small and moderate values of eccentricity. To be exact, expansions in elliptic motion apply to eccentricities which are less than 0.663 . Above that value, the series are no longer absolutely convergent. King-Hele used the equation, truncated to the order of $e^{3}$ and showed that his theory is accurate for orbits with $e$ less than $\mathbf{0 . 2}$. Because of the difficulty he encountered in the analytic integration, he divided the contraction of the orbit into two phases. For phase 1 , the eccentricity has the approximate range $0.02<e<0.2$. In this phase the range of $x$ is $3<x<30$ and the integration is performed using the asymptotic form of the Bessel ratios $y_{i}(x)$. For phase 2, the eccentricity has the approximate range of $0<e<0.02$, and the range for $x$ is $0<x<3$. Simplification by asymptotic expansion is not available, but integration to the first order in $e$ is feasible. Also, for the case of large values of $x$, the integration by King-Hele involves a heuristic step in that he assumes a certain form for the solution $Z$ on the right hand side of eqn (51) in its asymptotic form so that finally $Z$ can be obtained by quadrature.

For the present formulation, the nonlinear equation (51), to the order of $e^{4}$ has been integrated rigorously to the order of $\epsilon^{3}$. The artificial division of the contraction of the orbit into two phases has been removed and the solution is uniformly valid for the entire lifetime of the orbit for $e$ in the range $0<e \leq e_{0}$.

\section{Explicit formulas for the orbital elements}

For small and moderate eccentricity $(e<0.4)$, the solutions obtained, (eqns 66-70), are very accurate. This has been verified by computing the numerical integration of the nonlinear eqn (51) and its analytic solution, (eqns 66 and 67). In this case, the solution was always found to be greater than the numerical integration with a maximum error of approximately $c e_{0}^{3} / 5\left(1-e_{0}^{2}\right)$ for $0.1 \leq e_{0} \leq$ 0.99. It is interesting to note that even as $e_{0} \rightarrow 1$, which is outside the region of strict mathematical validity, the maximum error is less than $1 / 10 \beta r_{m e}$ (of the order $10^{-3}$ ). For small values of eccentricity the solution is extremely accurate. For example, when $e_{0}=0.1$ and $\epsilon=0.008$, eqns (66) and (67) provide 7 digits of accuracy, while for the same case King-Hele's solution gives only 4 digits of accuracy. Thus, the present solution provides a major improvement in that it is much more accurate and that it is uniformly valid for all eccentricities, $0<e \leq$ $e_{0}$.

The solutions obtained for the orbital elements, $a / a_{0}, e / e_{0}$, etc. are expressed in terms of the variable $x$. It would be useful to derive formulas to relate any pair of orbital elements. This amounts to the elimination of $x$ between any two of the 
eqns (67-70). Because of the transcendental nature of the solutions, the task is cumbersome. Fortunately, since $\epsilon$ is small, elimination of $x$ through Lagrange's expansion is feasible.

To derive the explicit expression for the semi-major axis in terms of the eccentricity, we write eqn (67) as

$$
Z=p+\epsilon \phi(Z)
$$

where, by observing that

$$
x=\beta a e=\beta a_{0} e_{0}\left(\frac{a}{a_{0}}\right)\left(\frac{e}{e_{0}}\right)
$$

we can write

$$
x=\alpha Z
$$

with

$$
\alpha=x_{0}\left(\frac{e}{e_{0}}\right)
$$

Then, explicitly

$$
\phi(Z)=Z_{1}(\alpha Z)+\epsilon Z_{2}(\alpha Z)+\epsilon^{2} Z_{3}(\alpha Z)+\cdots
$$

If Lagrange's expansion is applied to eqn (71), we have

$$
Z=p+\sum_{n=1}^{\infty} \frac{\epsilon^{n}}{n !}\left(\frac{d}{d p}\right)^{n-1}[\phi(p)]^{n}
$$

If we carry out the expansion, and then put $p=1$, we shall have to the order of $\epsilon^{5}$

$$
Z=1+\epsilon h_{1}(\alpha)+\epsilon^{2} h_{2}(\alpha)+\epsilon^{3} h_{3}(\alpha)+\epsilon^{4} h_{4}(\alpha)+\epsilon^{5} h_{5}(\alpha)
$$

where

$$
\begin{aligned}
h_{1}= & Z_{1} \\
h_{2}= & 2\left(A-A_{0}\right)+(A-3) Z_{1} \\
h_{3}= & \frac{7}{2}\left(\alpha^{2}-x_{0}{ }^{2}\right)-\left(2 A_{0}+\frac{13}{2}\right)\left(A-A_{0}\right)+\left(13+2 \alpha^{2}-4 A-A^{2}\right) Z_{1} \\
& +\frac{1}{2}\left(3+\alpha^{2}+A-A^{2}\right) Z_{1}^{2}
\end{aligned}
$$




$$
\begin{aligned}
& h_{4}=-\frac{1}{2}\left(\alpha^{2}-x_{0}^{2}\right)\left(35+4 A_{0}-7 A\right) \\
& +\frac{1}{6}\left(A-A_{0}\right)\left(213+42 A_{0}+16 A_{0}^{2}+12 \alpha^{2}-9 A+4 A_{0} A-8 A^{2}\right) \\
& -\frac{1}{2} Z_{1}\left(138+25 \alpha^{2}-46 A-7 A^{2}-2 A^{3}\right) \\
& -\frac{1}{2} Z_{1}^{2}\left(35+7 \alpha^{2}-6 A^{2}+\alpha^{2} A-A^{3}\right) \\
& +2\left(A-A_{0}\right) Z_{1}\left(3+\alpha^{2}+A-A^{2}\right) \\
& -\frac{1}{6} Z_{1}^{3}\left(6-\alpha^{2}+2 \alpha^{2} A+3 A^{2}-2 A^{3}\right) \\
& h_{5}=-\frac{3}{4}\left(\alpha^{2}-x_{0}^{2}\right)^{2}+\frac{1}{8}\left(\alpha^{2}-x_{0}^{2}\right)\left(885+68 \alpha^{2}-92 A-12 A^{2}\right) \\
& -\left(\alpha^{2}-x_{0}^{2}\right)\left(A-A_{0}\right)\left(\frac{19}{2}+4 A_{0}+2 A\right) \\
& -\left(A-A_{0}\right)\left(\frac{441}{2}+\frac{33}{2} \alpha^{2}+\frac{137}{4} A+2 \alpha^{2} A+\frac{9}{2} A^{2}+2 A^{3}\right) \\
& +\left(A-A_{0}\right)^{2}\left(\frac{409}{8}+2 \alpha^{2}+23 A+12 A^{2}\right)-\left(A-A_{0}\right)^{3}\left(10+\frac{40}{3} A\right) \\
& +4\left(A-A_{0}\right)^{4}+\frac{7}{2}\left(\alpha^{2}-x_{0}^{2}\right) Z_{1}\left(3+\alpha^{2}+A-A^{2}\right) \\
& +Z_{1}\left(437+88 \alpha^{2}+2 \alpha^{4}-154 A-5 \alpha^{2} A-22 A^{2}-3 A^{3}-A^{4}\right) \\
& -\frac{1}{2}\left(A-A_{0}\right) Z_{1}\left(143+29 \alpha^{2}+25 A+12 \alpha^{2} A-17 A^{2}-12 A^{3}\right) \\
& +2\left(A-A_{0}\right)^{2} Z_{1}\left(3+\alpha^{2}+A-A^{2}\right) \\
& +\frac{1}{4} Z_{1}^{2}\left(648+133 \alpha^{2}+4 \alpha^{4}-96 A+14 \alpha^{2} A-91 A^{2}-4 \alpha^{2} A^{2}-10 A^{3}\right) \\
& -\left(A-A_{0}\right) Z_{1}^{2}\left(6-\alpha^{2}+2 \alpha^{2} A+3 A^{2}-2 A^{3}\right) \\
& +\frac{1}{6} Z_{1}^{3}\left(123+4 \alpha^{2}-\alpha^{4}-6 A+18 \alpha^{2} A+15 A^{2}+2 \alpha^{2} A^{2}-18 A^{3}-A^{4}\right) \\
& +\frac{1}{24} Z_{1}^{4}\left(18-\alpha^{2}-2 \alpha^{4}-8 \alpha^{2} A-3 A^{2}+8 \alpha^{2} A^{2}+12 A^{3}-6 A^{4}\right) .
\end{aligned}
$$

In the expressions for $h_{i}(\alpha)$, we have defined

$$
A=\alpha y_{0}(\alpha), \quad Z_{1}=\log \frac{\alpha I_{1}(\alpha)}{x_{0} I_{1}\left(x_{0}\right)}
$$

Since $\alpha=x_{0}\left(e / e_{0}\right)$, the solution, as given by eqns (76) and (77) provides an explicit expression of the variation of $a / a_{0}$ as a function of $e / e_{0}$.

The other orbital elements can also be expressed in terms of $e$. The drop in 
the periapsis is

$$
\frac{r_{p_{0}}-r_{p}}{H}=\left(\alpha-x_{0}\right)-(1-e)\left(h_{1}+\epsilon h_{2}+\epsilon^{2} h_{3}+\epsilon^{3} h_{4}+\epsilon^{4} h_{5}\right) .
$$

For the apoapsis, we have

$$
\frac{r_{a_{0}}-r_{a}}{H}=\left(x_{0}-\alpha\right)-(1+e)\left(h_{1}+\epsilon h_{2}+\epsilon^{2} h_{3}+\epsilon^{3} h_{4}+\epsilon^{4} h_{5}\right) .
$$

In addition, the following formulas can be easily derived

$$
\begin{aligned}
& \frac{r_{p}}{r_{p_{0}}}=\frac{(1-\epsilon \alpha)}{\left(1-e_{0}\right)} Z(\alpha) \\
& \frac{r_{a}}{r_{a_{0}}}=\frac{(1+\epsilon \alpha)}{\left(1+e_{0}\right)} Z(\alpha) \\
& \frac{T}{T_{0}}=Z^{3 / 2}(\alpha) .
\end{aligned}
$$

The last expression provides the orbital period as a function of the eccentricity. As pointed out by King-Hele, such a relationship, if it is accurate, which is the case in the present theory, provides a powerful method of verifying the assumption made on the atmosphere from two of the most accurate and easily measured orbital parameters, namely, the period of revolution and the eccentricity.

The contraction of highly eccentric orbits

For the case of orbits with large eccentricities, King-Hele has used an entirely different method to derive the basic equation for the contraction. Using the present notation, we have his equation in the form

$$
\frac{d Z}{d x}=\epsilon\left(1+\frac{1}{2 x}\right)-\frac{\epsilon^{2}}{Z+\epsilon x}
$$

It is possible to obtain this equation from the basic eqn (51). Since $x=\beta a e$, when $e \rightarrow 1, a \rightarrow \infty, x$ becomes very large and the asymptotic expression for Bessel's ratio $y_{0}(x)$ is

$$
\begin{aligned}
& y_{0}=1+\frac{1}{2 x}+\cdots \\
& y_{0}^{2}=1+\frac{1}{x}+\cdots
\end{aligned}
$$

Using this form in eqn (51) we have 


$$
\frac{d Z}{d x}=\epsilon\left(1+\frac{1}{2 x}\right)-\frac{\epsilon^{2}}{Z}+\frac{\epsilon^{3} x}{Z^{2}}-\frac{\epsilon^{4} x^{2}}{Z^{3}}+\frac{\epsilon^{3} x^{3}}{Z^{4}}-\cdots
$$

an equation which can be seen as the development of eqn (84). While King-Hele can only provide an approximate solution to the nonlinear eqn (84) by assuming that on the right hand side $Z$ is approximated by $Z=1-\epsilon\left(x_{0}-x\right)$ so that the equation can be integrated by simple quadrature, it is enlightening to know that the exact solution to the equation can be obtained. This is done by using the transformation

$$
Z=\epsilon(x+q)
$$

and changing the independent variable from $x$ to $q$ to have the Bernoulli equation

$$
\frac{d x}{d q}=2 x+\frac{4 x^{2}}{q}
$$

Using the change of variable,

$$
x=\frac{\mathrm{e}^{2 q}}{K(q)}
$$

we have after substituting into (88)

$$
\frac{d K}{d q}=\frac{-4 e^{2 q}}{q}
$$

Integrating, we find that $K$ can be expressed in terms of the exponential integral.

$$
\begin{aligned}
K & =-4 \int \frac{\mathrm{e}^{2 q}}{2 q} \mathrm{~d}(2 q)+C \\
& =-4 E_{l}(2 q)+C .
\end{aligned}
$$

Thus, we have the exact solution in parametric form.

$$
x=\frac{\mathrm{e}^{2\left(q-q_{0}\right)}}{\frac{1}{x_{0}}+4 \mathrm{e}^{-2 q_{0}}\left[E_{i}\left(2 q_{0}\right)-E_{i}(2 q)\right]}
$$

along with eqn (87) and the initial conditions

$$
\begin{aligned}
Z\left(x_{0}\right) & =1 \\
q_{0} & =\frac{1}{\epsilon}-x_{0}=\frac{\left(1-e_{0}\right)}{\epsilon} .
\end{aligned}
$$


To evaluate the exponential integral, we first observe that the argument $2 q$ is large, hence its asymptotic form is adequate. In general, we consider the integral

$$
E_{n}(x)=\int e^{x} x^{n-1} \mathrm{~d} x
$$

By integration by parts

$$
E_{n}(x)=x^{n-1} e^{x}-(n-1) E_{n-1}
$$

By repeated application of this formula, we deduce the asymptotic expansion for large $\boldsymbol{x}$

$$
E_{n}(x)=x^{n-1} e^{x}\left[1-\frac{(n-1)}{x}+\frac{(n-1)(n-2)}{x^{2}}-\frac{(n-1)(n-2)(n-3)}{x^{3}}+\cdots\right]
$$

When $n=0$, we have the exponential integral and by taking 6 terms of the series, for $x>50$, the solution is identical to the numerical values tabulated by Abramowitz and Stegun (1972).

\section{Numerical application}

The theory applies to any planetary atmosphere that is locally exponential. Numerical examples have been selected for a wide range of orbits with the values of $H$ appropriate for the Earth's atmosphere assumed spherical. The numerical solution is obtained by integrating the basic nonlinear eqn (51). The analytic solution employed is either the solution $Z=Z(x)$ as given by eqns (66) and (67) or the explicit solution $Z=Z(\alpha)$ as given by eqns (76) and (77).

The parameters used are the initial eccentricity $e_{0}$ and the initial perigee distance $r_{p_{0}}$, or equivalently the dimensionless small parameter $1 / \beta r_{p_{0}}$. Then we have

$$
\epsilon=\frac{\left(1-e_{0}\right)}{\beta r_{p_{0}}}
$$

which tends toward zero as $e_{0} \rightarrow 1$. By using the three values $1 / \beta r_{p_{0}}=0.005,0.01$ and 0.02 we cover a wide range of perigee heights.

Figure 3 plots the variation of $Z=a / a_{0}$ as function of $x / x_{0}$ for different values of the eccentricity. Since the analytical solution has a high degree of accuracy, its small deviation in the fifth or sixth digit from the numerical solution cannot be detected in the figure. Initially the solution is nearly linear with the slope in the figure approximately equal to $e_{0}$, but near the end, as $x$ and $e$ approach zero, it exhibits rapid decay. This explains the difficulty encountered by King-Hele in his analytic integration. It is interesting to note that the analytic solution $Z(x)$ of the truncated eqn (51) remains accurate for high eccentricity. For $e_{0}=0.99$, this solution and the exact solution (87) and (92) of the asymptotic eqn (84) are nearly identical except for very small values of $x / x_{0}$. 


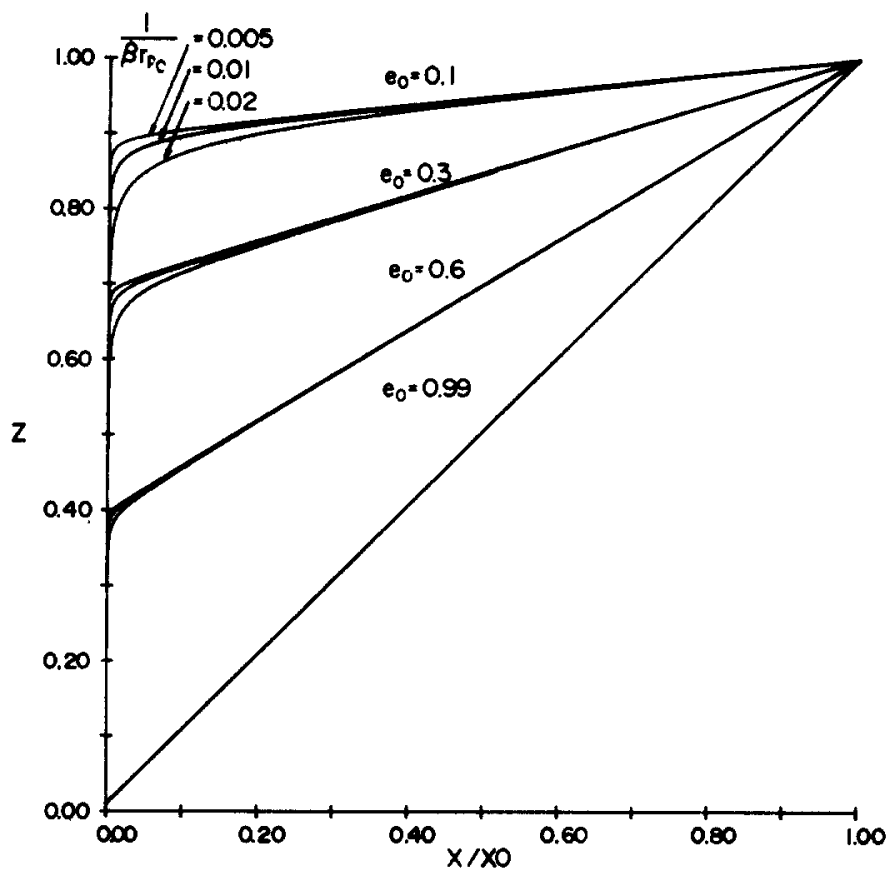

Fig. 3. Variations of $Z=a / a_{0}$ as function of $x / x_{0}$.

Figure 4 plots the solution $Z=Z(\alpha)=Z(e / \epsilon)$ as a function of the eccentricity for several values of $e_{0}$. The range of validity is limited to $e_{0}=0.5$ since the $Z(\alpha)$ solution is not as accurate as the $Z(x)$ solution. It was found that $Z(\alpha)$ exceeds the numerical solution by a maximum value approximated by $e_{0}^{6} / 15\left(1-e_{0}\right)$, which still gives 7 digits of accuracy for $e_{0}=0.1$, but diverges as $e_{0} \rightarrow 1$. For $e_{0}=0.5$ the error is imperceptible in the figure. The decay in the perigee distance $r_{p}$ versus the eccentricity for $e_{0}=0.1$ and $e_{0}=0.4$ is presented in Fig. 5 . The ratio $r_{p} / r_{p 0}$ remains nearly equal to one for a large portion of the decay process, but as $e \rightarrow 0$ the drop in perigee altitude increases rapidly. For small values of $1 / \beta r_{p 0}(0.005)$ the decay is much slower than for large values $(0.02)$ as evident in the figure. The fractional error in this plot and in the next two is kept to an imperceptible amount by considering the error formula mentioned earlier.

The decay in the apogee distance $r_{a}$ versus the eccentricity for several values of $e_{0}$ is presented in Fig. 6. It is evident that the ratio $r_{d} / r_{a_{0}}$ decreases rapidly with the eccentricity. Initially the parameter $1 / \beta r_{p_{0}}$ seems to have little effect, but as the eccentricity approaches zero the larger values of $1 / \beta r_{p 0}$ yield more rapid decay.

Finally the decay in the orbital period $T$ as function of the eccentricity is presented in Fig. 7. As pointed out by King-Hele this functional relationship $T=T(e, \epsilon)$ provides a powerful formula for testing the atmospheric parameter $\epsilon$ since orbital period and eccentricity can be accurately measured. 


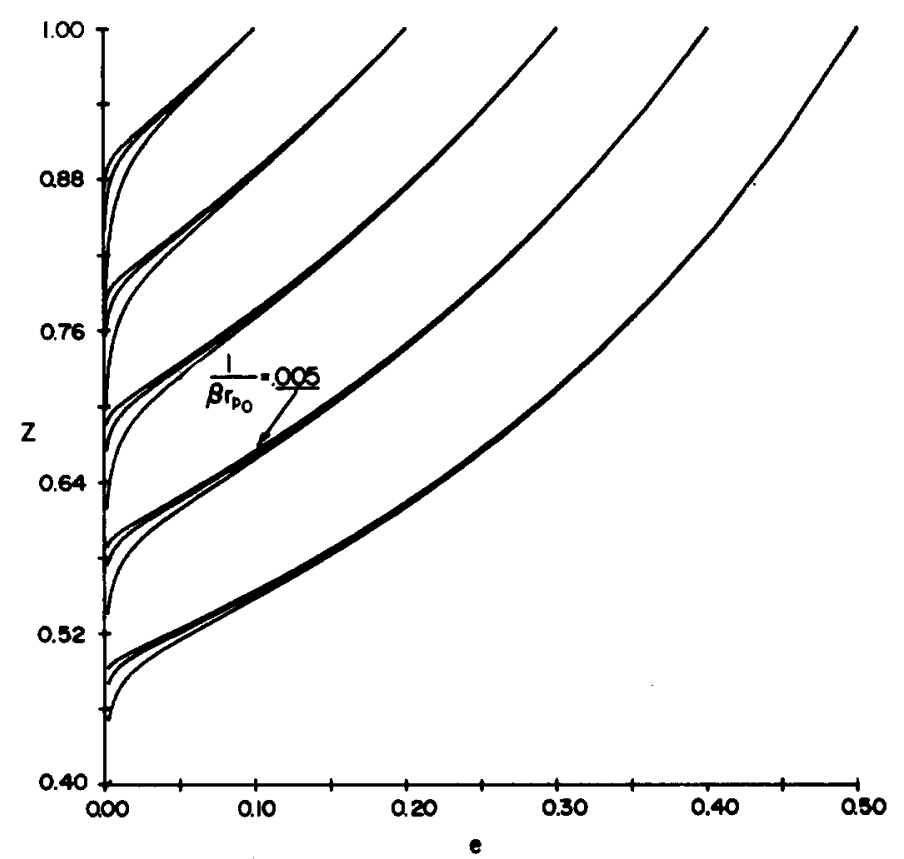

Fig. 4. Variations of $Z=a / a_{0}$ as function of $e$.

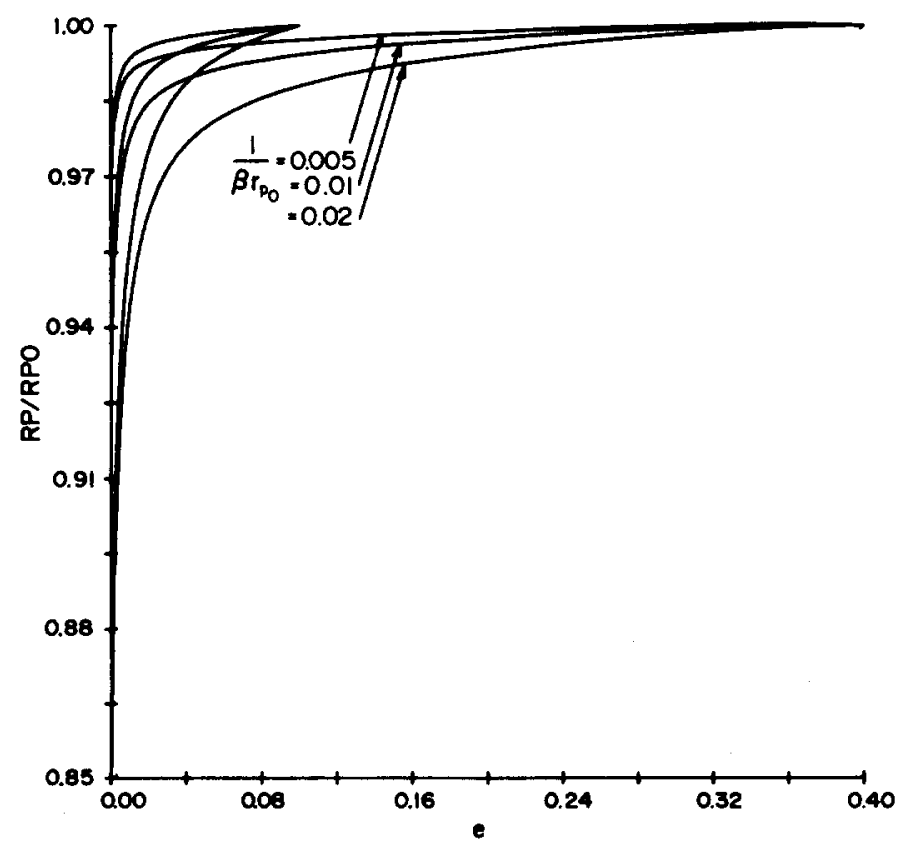

Fig. 5. Variations of the perigee distance. 


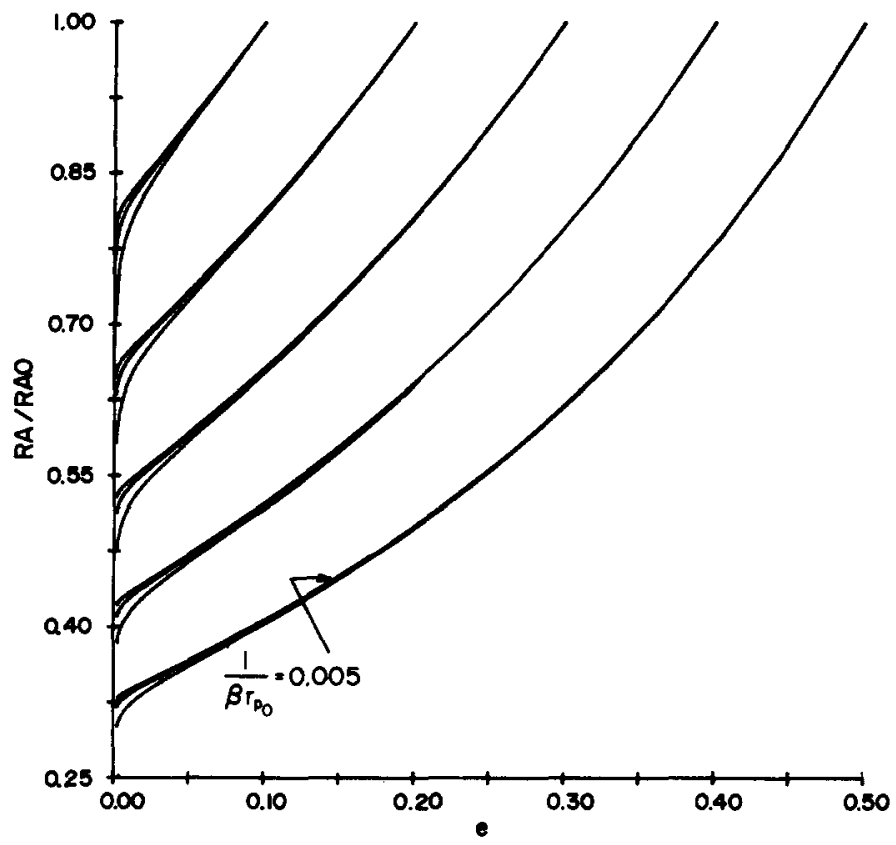

Fig. 6. Variations of the apogee distance.

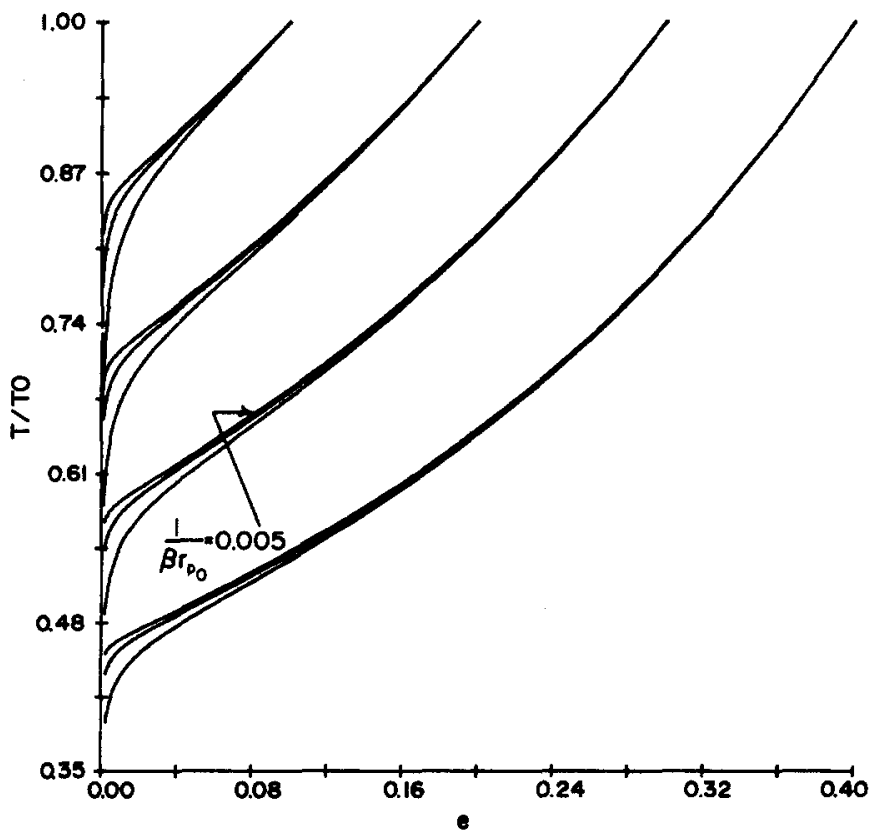

Fig. 7. Variations of the orbital period. 


\section{Conclusions}

To bridge the gap between satellite theory in the presence of an atmosphere and entry theory we have developed a set of equations using a set of dimensionless variables applicable to both cases. For a first estimate of physical quantities like the altitude, the speed, the deceleration and the heating rate during entry, a spherically symmetrical atmosphere with exponential variation of density with height seems to be adequate. For satellite theory there are several perturbing factors that should be taken into consideration. For example, for orbits with eccentricities greater than $\mathbf{0 . 5}$, the lunisolar perturbations cannot be neglected. For orbits with smaller eccentricities, the theory can only be accurate if a realistic model for the atmosphere is employed. For the sake of mathematical purity of the solution we have adopted a spherical atmosphere which is locally exponential. In this respect, the scale height $H$ varies with the altitude, so that different values of $\beta$ have to be used at difierent phases of the satellite's life. Also, it should be remembered that in the Earth's atmosphere, an oblateness of $20 \mathrm{~km}$ can greatly alter the density at any distance $r$. This has been discussed by King-Hele (1964) and, following his development, we can use the method of integration as presented in this paper to generate the additional perturbing terms to the solution which account for the effects of the oblateness.

\section{References}

Abramowitz M. and Stegun I. A. (1972) Handbook of Mathematical Functions. Dover Publications, New York.

Bogoliubov N. N. and Mitropolaky Y. A. (1961) Asymptotic Methods in the Theory of Nonlinear Oscillations. Gordon Breach, New York.

King-Hele D. (1964) Theory of Satellite Orbits in an Atmosphene. Butterworths, London.

Moulton F. R. (1920) Periodic Orbits. Carnegie Foundation XXV No. 162.

Poincare H. (1960) Les MCthodes Nouvelles de la Mecanique CAleste, Vol. 1. Dover Reprint.

Vinh N. X., Busemann A. and Culp R. D. (1975) Optimum three-dimensional atmospheric entry. Acta Astronautica 2(7/8), 593-611.

Vinh N. X., Blestos N. A., Busemann A. and Culp R. D. (1977) Flight with lift modulation inside a planetary atmosphere. AIAA J. 15(11), 1617-1623. 